\title{
PRACTICAL TRAINING OF ROBOTIC CONCEPTS USING INTERACTIVE TOOLS
}

\author{
Carlos Alberto Jara Bravo, Francisco Andrés Candelas Herías, Fernando Torres Medina
}

\author{
University of Alicante \\ Crta San Vicente del Raspeig $s / n$ \\ PO Box 03080, San Vicente del Raspeig-Spain \\ cajb@dfists.ua.es,Francisco.Candelas@ua.es,Fernando.Torres@ua.es
}

\begin{abstract}
This paper presents an interactive computer simulator that can be used in the field of practical training in Robotics. The tool can provide basic training for workers as well as increase their knowledge about robotic concepts. The application offers a very user-friendly interface and a realistic environment which allows the practice of many robotic principles. It also introduces Easy Java Simulations (EJS for short), a Java-based tool which has been used to create the virtual laboratory. Copyright (C) 2007 IFAC
\end{abstract}

Keywords: robotics; training; virtual reality; simulation; programming.

\section{INTRODUCTION}

With the knowledge evolution and the appearance of new technologies, as for example robotic systems, it is necessary to use qualified people for the effective use of its sources in order to improve its usefulness. So, formation and training of workers is becoming an essential need for the strategy company.

Robotic systems are often complex and difficult to operate. In addition, training the operators and programmers of these complex robotic systems is time consuming and costly. It also is possible that the robotic equipment can be damaged if it is used improperly. Nowadays, new technologies such as virtual reality allow the operator to be trained to operate complex robotic systems in an intuitive and cost-effective way.

The use of interactive tools or virtual laboratories for instruction is particularly significant because it provides practical insight into robotic fundamentals. So, a virtual laboratory has been developed for training in robotics which allows operators to interact with simulations of real robots in an intuitive and natural way. It makes possible an early introduction of robotic concepts such as mechanic structure (links, joints), Denavit-Hartenberg parameters, direct and inverse cinematic and path planning. Thus, it is possible to simulate the robot trajectory of a task and to test the robot behaviour. It also offers a user interface which is very user-friendly for operators who are not specialist in Robotics.
The paper is structured as follows. First, the importance of virtual labs for robotic control in the industry is explained. The following section describes basic aspects of EJS, a Java-based tool intended to create interactive simulations. Afterwards, Section 4 explains the principal features of the virtual laboratory developed. The actual and future work lines are described in Section 5. Finally some important conclusions are shown in Section 6.

\section{VIRTUAL LABORATORIES FOR ROBOTIC CONTROL}

Robots have become important in a wide range of applications from manufacturing (Vazquez, et al., 2006) to surgery (Thorne, et al., 1995), in tasks like the handling of hazardous materials (Sabater, et al., 2006), high precision tasks, etc. Nowadays, the robotic systems allow to increase the productivity flexibility and every time they are more frequently used in the industry. Consequently, it is important to understand how they work, and to have qualified people to manipulate them.

The creation of virtual training environments can provide adequate robotic learning elements for the operators. They simulate the devices, including all of the required parameters, so that the operator could work with a complete and realistic robotic environment. Therefore, virtual laboratories for robotic control offer a new solution to meet their "hands-on" learning needs and to acquire high-level skills to control the robot. In addition, with the 
development of the Web as a viable medium for learning and training in a more flexible and customer-aware manner (Dormido, 2004), the virtual laboratories allow the operator to carry out practical exercises from other places (like at home).

At present, there are a lot of research groups about virtual reality and virtual laboratories. They develop the interactive tools to move the human-robot interface to a new, intuitive and user-friendly level. So, there are a lot of virtual laboratories developed in the field of robotic systems (Candelas and Moreno, 2005). This fact shows the significance of these applications both learning and industry.

Moreover, the virtual labs are used in the robot industry to reduce training delivery costs, increase productivity and accelerate proficiency and retention.

In spite of large amounts of tools developed, the new virtual laboratory presented here provides some features such as: it is based on the portable language Java, it offers an open architecture and it is possible to manipulate a lot of robotic parameters, which only a few robotic applications have. Authors try to improve a virtual and remote laboratory developed in their research group and called Robolab (Candelas, et al., 2003), whose success in student teaching has been proved (Torres, et al., 2006).

\section{EASY JAVA SIMULATIONS FUNDAMENTALS}

Virtual laboratories have been used in the industry for many years. These provide workers tools for exploring and testing the system, and can help them to understand some complex concepts through simulation. However, creating a computer simulation requires an extra effort because it needs the programming and technical expertise to describe the system in computer language. With EJS, the task of creating a computer simulation is greatly simplified.

EJS is an open-source tool developed in Java language designed for the creation of interactive virtual labs (Esquembre, 2004). EJS was developed for an Open Source Physics Project which was established to create and distribute curricular material for physics computation (Christian and Belloni, 2003). So, the computer simulations created with EJS can be used as stand-alone Java program under different operations systems or be distributed via the Internet as applets.

The principal reason to choose EJS to create our virtual lab is its simplicity of use and that it is not necessary to have specific programming skills (Dormido, et al., 2005). Therefore, there has not been needed a big investment of time to create an interactive graphical user interface and it has allowed us to concentrate most of our time on the description of the robotic system.
EJS is based in the successful model-view-control software paradigm, which states that a simulation is composed in three parts: the model, the view, and the control. The model describes the system in terms of variables (states of the system) and relationships of these variables (mathematical equations). It is the scientific part of the computer simulation. The view is the graphical representation of the different states that the system can have. The control is used to send messages to the model and provides the interface between the model and the interactive user interface devices (e.g. keyboard, mouse) (Krasner and Pope, 1988). The three parts are interconnected. A change in the state of the model affects the graphical representation of the view. The control messages modify the value of variables of the model. Finally, the view affects the model and the control because it is possible to modify variables of the model or to perform some control actions from the graphical user interface

EJS implements a simplification in the model-viewcontrol paradigm suppressing the control part, merging it half into the view, half into the model. Model and view are interconnected because it is possible to link model variables and view properties. So, there is a bi-directional communication between the view and the model. Therefore, any change in the model is displayed by the view, and any user interaction with the view modifies the value of the corresponding model variable.

The process to create an application with EJS includes the definition of the model and the view. With regard to the model, the user must declare the variables that the system describes, must initialize them to initial value and must write the equations that state how of these variables change in time or under user interaction. For this last step, EJS offers two options. The first one is an editor and solver of ordinary differential equations (ODE) where the user can write his/her equations and EJS generates the code that solves the system using one of several of the provided standard algorithms (Euler-Richardson, Runge-Kutta, etc,...) (Christian and Belloni, 2003). The second one is a connection to Matlab/Simulink that lets users specify and solve their models with the help of these tools (Sanchez, et al., 2005). So, the model can be defined with Matlab code in a Matlab function (M-file) or with a Simulink block diagram or with both. With regard to the view, EJS provides a set of standard Java Swing components and specific two and three dimensional visualization classes from the Open Source Physics Project to build the interface in a simple drag and drop way. These graphical components have certain properties that the user can connect them with the model variables and to set a link between the model and the view. So, the simulation turns into an interactive application.

After finishing the model and view, EJS generates the Java source code, compiles the source and creates an interactive simulation which can be run as a standalone Java program or as an applet in an HTML page. 


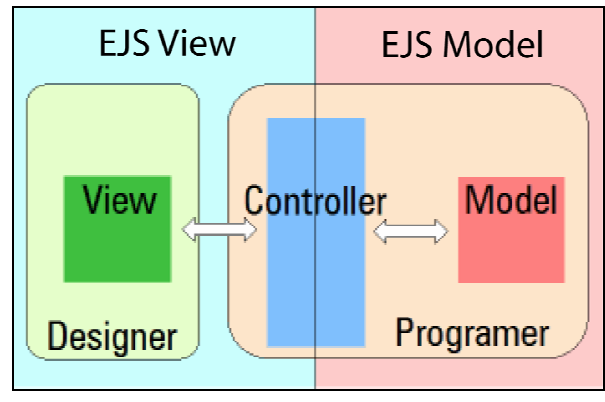

Fig. 1. EJS Paradigm

Finally, although there are a lot of virtual labs developed with EJS, up to now there was fewer in the field of industrial robot arms and generally they was not very complete.

\section{THE VIRTUAL LABORATORY DEVELOPED}

In automation, the use of new techniques requires both a basic and a specialized training for operators. Traditional methods and tools employed are usually expensive. The main goal of the developed tool is to offer a low cost solution for operators training. The need requirements to run the tool are to have a Pentium II $266 \mathrm{MHz}$ with $64 \mathrm{Mb}$ RAM o higher, to have installed the JVM (Java Virtual Machine) and to have installed Matlab 5.3 or higher.

According to the previous section, to create our virtual laboratory using EJS, it is necessary to perform the model of the robotic system and to build the view of the user interface. In order to develop the model, the connection of EJS with Matlab is used. The mathematical model of the system is defined by a series of M-files. Therefore, EJS is only used to develop the user interface and to declare the variables. These EJS variables are connected with the Matlab variables which the M-files generate. So, every change in the user interface is sent to the Matlab workspace and read by the M-files. At the same time, the outputs variables of M-files are written in the workspace read by EJS and used to refresh the simulation. Matlab workspace is the buffer that EJS and the code of M-files use to exchange data (Figure 2).

The view of the virtual laboratory developed has a main window, which is presented in Figure 3. The upper part contents a $3 \mathrm{D}$ representation of the workspace where the robot of six degrees of freedom is displayed. This part of the tool shows the graphical representation of the robot movement. At the bottom of the main window, there is a control panel with a set of sliders to move the robot (see 4.1). Finally, to the right, there are several controls with more options such as zoom, show/hide solids and show/hide D-H reference systems. In the next subsections, the virtual lab possibilities will be described better.

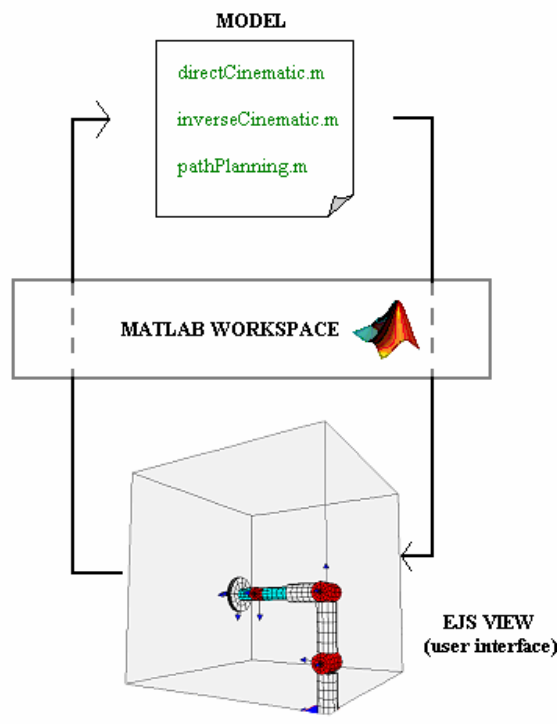

Fig. 2. Data flow between EJS and Matlab

\subsection{Direct and inverse cinematic.}

With the implementation in the application of the direct and inverse cinematic, the robot movement simulation is achieved. It is possible to move the robot with a joint control giving values at each one of the joints (sliders q1...q6) or with a cartesian control giving values at the end point of the robot (sliders $\mathrm{X}$, $\mathrm{Y}, \mathrm{Z}$ ). With regard to the cartesian control, it is possible to choose the options "elbow up" or "elbow down" (see right control in Figure 3). The interface also displays the transformation matrix with the position and the orientation of the end point and the values of the robot joints.

\subsection{Denavit-Hartenberg parameters.}

The Denavit-Hartenberg parameters are values that define the robot geometry. With the virtual lab, it is possible to edit and to change these values. There is a specific dialog control with several sliders to do it. In addition, it is possible to change the link radius (Figure 4). The graphical representation is updated dynamically while the parameters are changed, and therefore, it is very illustrative.

\subsection{Path planning.}

The model of the system implements a path planning. The formulation of the 4-3-4 polynomial trajectories, which generates a smooth robot movement, is used. This path planning divides the robot trajectory in three parts: actuator acceleration, time of maximum actuator velocity and actuator deceleration. 


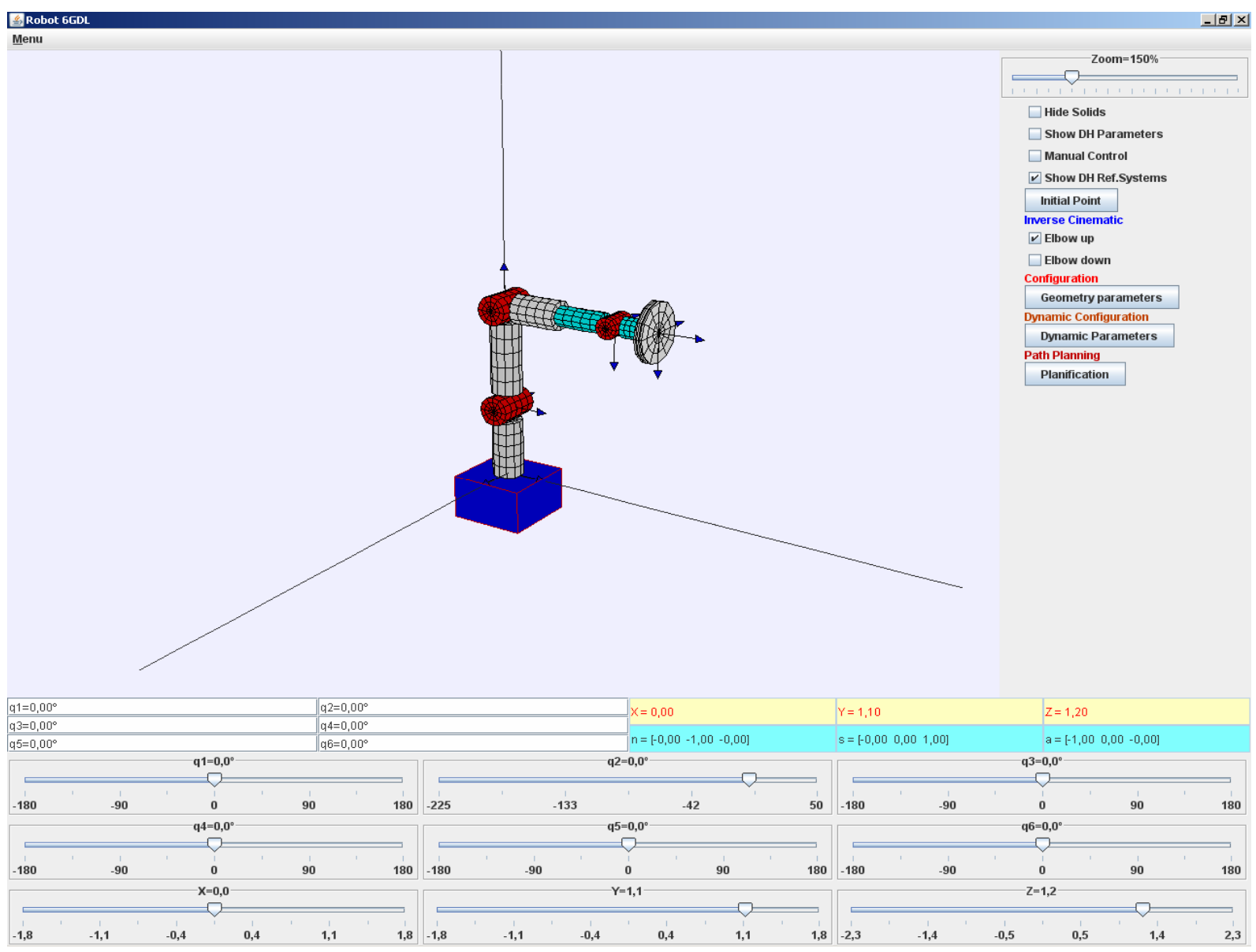

Fig. 3. User Interface.

To make a correct path planning, the user must introduce the following values: acceleration and deceleration time, maximum joint velocities, the initial and the end point. With regard to the values of the initial/end points, it is possible to introduce to them both joint and cartesian coordinates. In addition, the application plots the 3D trajectory in the workspace, the temporal evolution of joint values, joint velocities and joint accelerations (Figure 5). After finishing the trajectory, the interface has the possibility to save it. The trajectories are showed in a text area and it is possible to execute them at any time.

\subsection{Main results.}

The use of EJS involves several practical advantages as follows. The development of a virtual lab with EJS is very simple. Authors have employed few days to develop the presented here. The interface obtained is very similar to others developed with EJS (Figure 6). In addition, the application developed can be integrated with other applications created with EJS in an environment such as eMersion (Gillet, et al., 2005), a software tool which supports the management of these elearning systems.
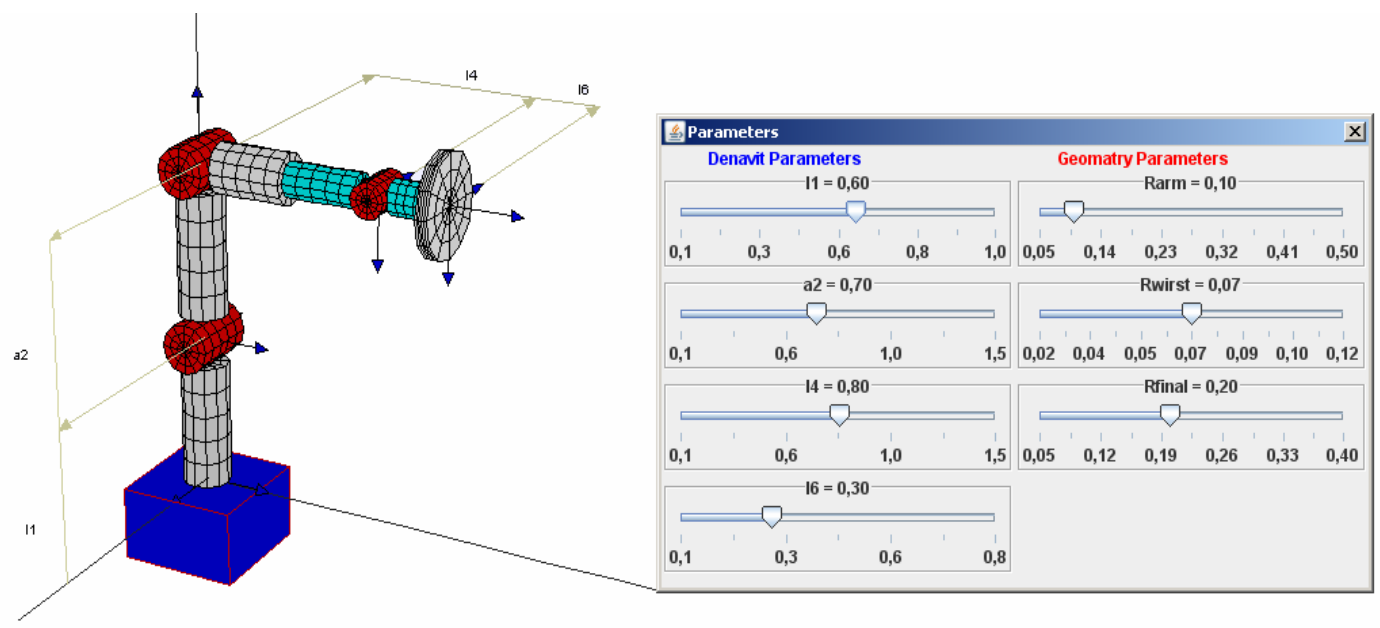

Fig. 4. Geometry Parameters. 


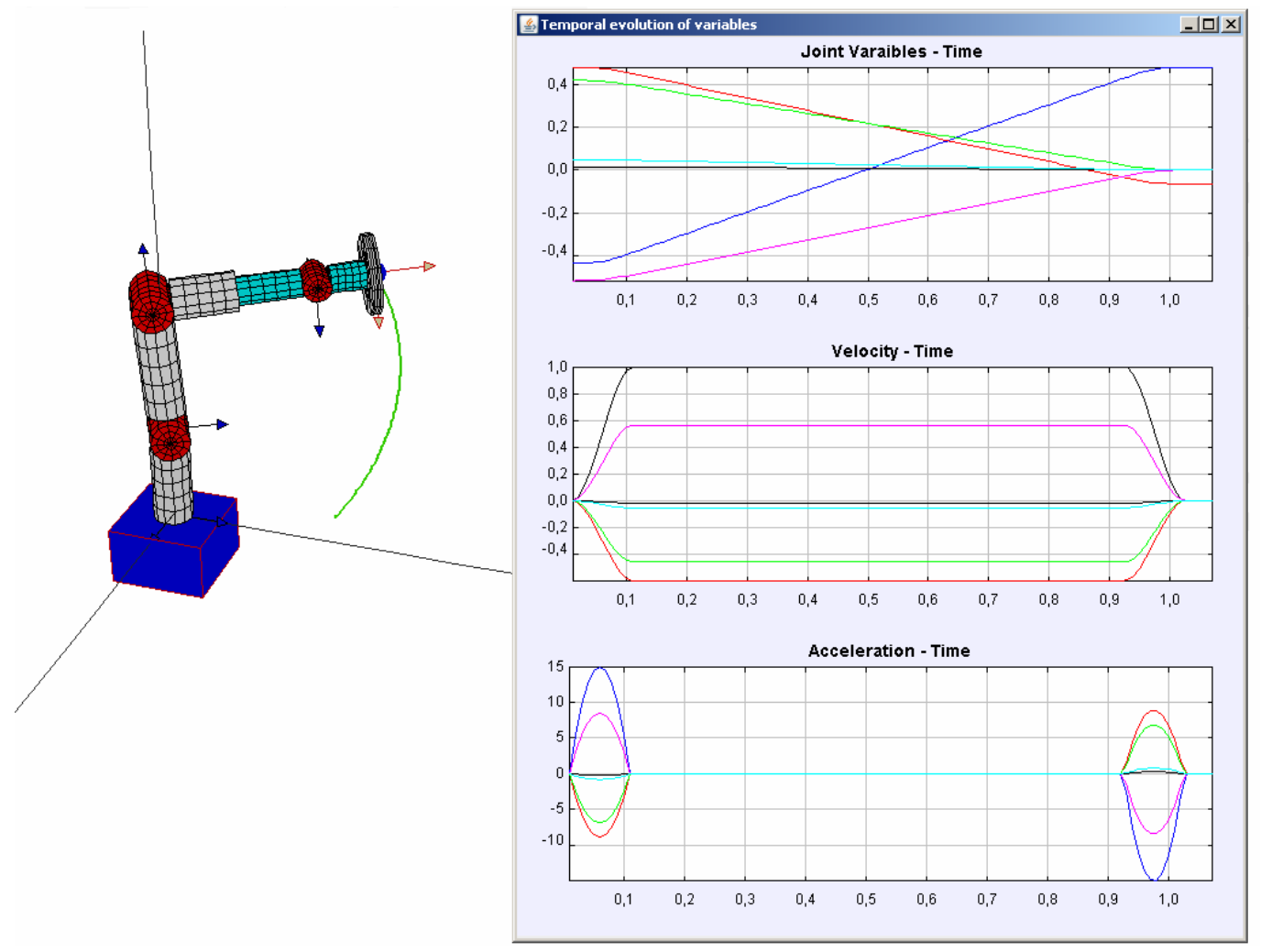

Fig. 5. Path Planning

\section{ACTUAL AND FUTURE WORKING LINES}

At present, authors continue developing the application described here and are working on including new features to both the virtual lab and the robotic system simulated.

With regard to the virtual lab, the first feature is to offer the possibility to add new robots of different characteristics in the workspace, due to the actual system that only permits to train a robotic system of six degrees of freedom. So, the user will be able to train with different robots in a more realistic virtual environment. Another feature is to improve the virtual laboratory with remote operation capabilities. Therefore, the user will be able to carry out operations with real robots through teleoperation. In this way, after training and testing movement commands with the virtual laboratory, it will be able to execute them remotely in the real robot.

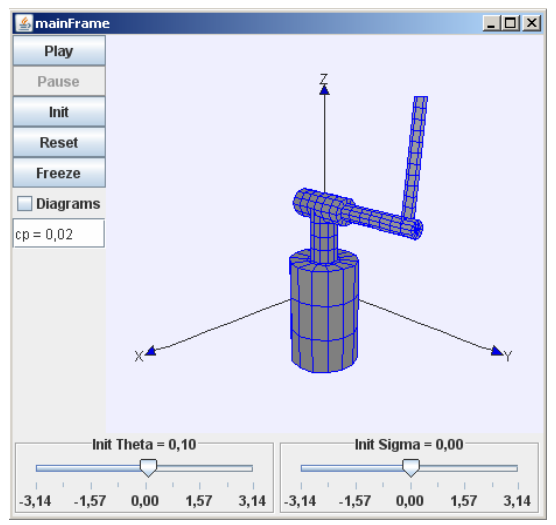

Fig. 6. Example of EJS Interface.
With regard to the robotic system, the main feature to add is the dynamic model. The actual system only includes the direct/inverse cinematic and path planning. With the dynamic model of the robotic system, the application will be a completely training tool. So, the user will be able to evaluate the torques in the actuators when the virtual lab is simulating a task.

After adding the features explained above, the application can be improved with new paths such as 3D recognition of basic objects in the workspace, the inclusion of virtual objects using augmented reality, an interpreting operator voice commands and audio feedback to guide the operator in the task.

\section{CONCLUSIONS}

In this paper, a virtual laboratory for the simulation of industrial robot arms has been presented. Our system is mainly oriented to the training of robotic concepts. The employment of virtual laboratories in the instruction field allows workers to be trained in a cost-effective way. In addition, they can be trained before the actual robot hardware is available or when physical robots are out of the loop. So, they are much better prepared to manipulate and to solve problems of complex robotic systems.

The application has been developed using EJS, an open-source tool designed for the creation of interactive simulations. So, the procedure to transform the robotic system in an interactive virtual laboratory has been easier to do than the 
majority of programs available. It has not been necessary to learn specific programming skills and a big investment of time has not been needed to create the application.

With the virtual lab developed, operators can learn robotic concepts such as direct/inverse cinematic and path planning. The user interface is very userfriendly, and the graphical simulation very realistic. In addition, EJS generates the applet simulation with the advantage that the operators can run the application from other place in free schedules.

Finally, the virtual laboratories generate an environmental simulation with which the user interaction is more intuitive and realistic. With these virtual tools, the employment training plan has a lot of quality and the company competence improves in the field of research and development.

\section{ACKNOWLEDGMENTS}

The work presented in this paper is supported by the "Ministerio de Educación y Ciencia" of the Spanish Government through FPI grants program (C.A. Jara) and research project DPI2005-06222. Authors would like to thank for this financial support.

\section{REFERENCES}

Candelas, F. A. and J. S. Moreno (2005). Recursos didácticos basados en Internet para el apoyo a la enseñanza de materias del área de Ingeniería de Sistemas y Automática. Revista Iberoamericana de Automática e Informática Industrial, 2, 93.

Candelas, F. A., S. T. Puente, F. Torres, F. G. Ortiz, P. Gil and J. Pomares (2003). A virtual laboratory for teaching robotics. International Journal of Engineering Education, 19, 363-370.

Christian, W. and M. Belloni (2003). Developing open source programs for science and mathematics. EUROCON 2003. Computer as a Tool. The IEEE Region 8, 1,

Dormido, S. (2004). Control Learning: Present and Future. Annual Reviews in Control, 28, 115-136.

Dormido, S., G. Farias, J. Sanchez and F. Esquembre (2005). Adding interactivity to existing Simulink models using Easy Java Simulations. Decision and Control, 2005 and 2005 European Control Conference. CDC-ECC'05. 44th IEEE Conference on, 4163-4168.

Esquembre, F. (2004). Easy Java Simulations: a software tool to create scientific simulations in Java. Computer Physics Communications, 156, 199-204.

Gillet, D., N. Anh Vu Nguyen and Y. Rekik (2005). Collaborative web-based experimentation in flexible engineering education. Education, IEEE Transactions on, 48, 696-704.

Krasner, G. E. and S. T. Pope (1988). A cookbook for using the model-view controller user interface paradigm in Smalltalk-80. Journal of Object-Oriented Programming, 1, 26-49.

Sabater, J. M., R. J. Saltarén, R. Aracil, E. Yime and J. M. Azorín (2006). Teleoperated parallel climbing robots in nuclear installations. Industrial Robot: An International Journal, 33, 381-386.

Sanchez, J., F. Esquembre, C. Martin, S. Dormido, S. Dormido-Canto, R. D. Canto, R. Pastor and A. Urquia (2005). Easy Java Simulations: an Open-Source Tool to Develop Interactive Virtual Laboratories Using MATLAB/Simulink. INTERNATIONAL JOURNAL OF ENGINEERING EDUCATION, 21, 798.

Thorne, G. C., M. Halliwell and P. N. T. Wells (1995). An experimental telesurgery robot. Towards Telesurgery, IEE Colloquium on, 4.

Torres, F., F. A. Candelas, S. T. Puente, J. Pomares, P. Gil and F. G. Ortiz (2006). Experiences with virtual environment and remote laboratory for teaching and learning robotics at the University of Alicante. International Journal of Engineering Education, 22, 766-776.

Vazquez, A. S., A. Adan, R. Torres and C. Cerrada (2006). Distributed control system of an experimental robotic cell with $3 \mathrm{D}$ vision. In: Icinco 2006: Proceedings of the Third International Conference on Informatics in Control, Automation and Robotics Robotics and Automation (Ed)), pp. 508511. 